\title{
A multi-animal model collaboration to speed up rare disease research
}

\author{
Elizabeth Doughman
}

F ive independent biomedical research organizations are teaming up in the fight to advance rare disease research, thanks to a $\$ 20$ million grant from the National Institute of Health's (NIH) National Center for Advancing Translational Sciences (NCATS).

The collaboration-made up of Lovelace Biomedical, The Jackson Laboratory, Exemplar Genetics, IONTOX, and the University of Pennsylvania-will rely on the individual strengths of each organization to form a complete pipeline to advance rare disease research. Member organizations will work together to establish novel animal models that will be used within the collaboration to help advance drug discovery and development for rare and neglected diseases.

The collaboration will also address important unmet research needs such as addiction and tropical infectious diseases.

"It's very difficult to find one organization that does it all, but when you look at the drug discovery pipeline, that's exactly what happens. You need toxicology data and proof of concept research in both small and large animal models. And that's what this collaboration is: several specialized areas coming together to create the complete package," said Cat Lutz, a research scientist and the senior director of the Mouse Repository at The Jackson Laboratory.

The Jackson Laboratory, a nonprofit biomedical research institution based in Bar Harbor, ME, will work to develop mouse models to be used in rare disease research, while researchers at the University of Pennsylvania in Philadelphia will focus on novel canine and feline options.

Minipigs bred at Iowa-based Exemplar Genetics will provide rare disease researchers with a large animal model to test the efficacy of new medications and devices. "The miniswine is similar to a human in metabolism, size and anatomy, making them a useful resource for drug discovery and preclinical efficacy testing," said Troy Arends, business director at Exemplar Genetics.

Albuquerque, NM-based Lovelace Biomedical will develop animal models in areas such as infectious diseases, addiction or pain, and will collaborate with the other

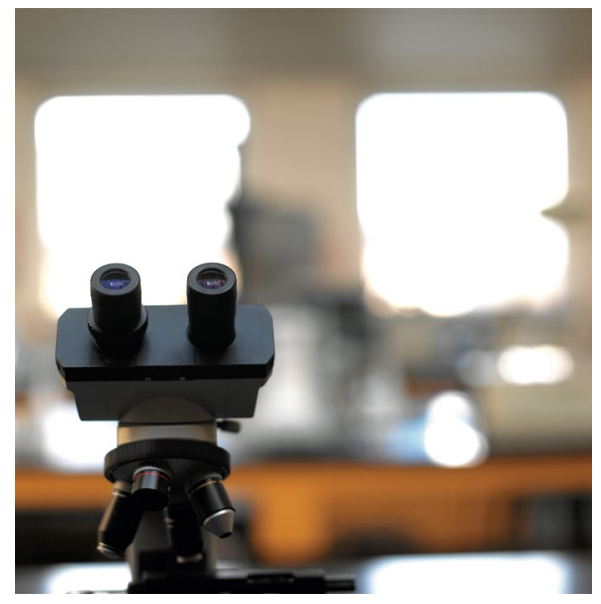

Credit: Stockbyte Royalty Free Photos

laboratories to help characterize the phenotype of the models in natural history studies.

Once scientists have developed one or more animal models with a rare disease phenotype or genotype, the two contract research organizations involved in the collaboration, Lovelace Biomedical and IONTOX from Kalamazoo, MI, will conduct preclinical toxicology, pharmacological and safety testing required by the United States Food and Drug Administration (FDA) before possible new therapies can move into clinical trials.

"Without the ability to mimic human disease and study specific interventions and the safety of those interventions, advancements are limited," said Jake McDonald, Chief Scientific Officer at Lovelace Biomedical and Principal Investigator of the overall contract. "Rare diseases impact millions of people and are often debilitating and poorly treated by current technologies."

The Orphan Drug Act of 1983, created by United States lawmakers to encourage companies to develop new drugs for rare diseases, defines a rare disease as a condition that affects fewer than 200,000 people.

More than 25 million Americans have been diagnosed with at least one of the 7,000 currently known rare diseases, NCATS' Genetic and Rare Disease Information Center (GARD) says. Over 95 percent lack an FDA-approved therapy or treatment.
Most rare diseases are caused by genetic mutations, typically first appearing early in life. The newly formed research pipeline will focus primarily on rare diseases where gene therapy has shown promise. New gene editing techniques, such as CRISPR/Cas9, use engineered nucleases-also known as 'molecular scissors' - to delete, replace, or insert specific DNA sequence located within a living organism's genome. Recent advancements in gene editing techniques make such tools faster and easier to use.

Gene therapy could one day help patients with rare diseases caused by genetic mutations, such as sickle cell disease, spinal muscular atrophy, and Friedreich's Ataxia. However, gene editing techniques remain controversial and are currently limited to use in animal models until further safety and ethical guidelines have been established.

"In vivo studies are critical in rare disease drug discovery to understand if a candidate therapeutic under preclinical development has efficacy and engages the target/signaling pathway as designed, and for evaluating preliminary toxicity and safety parameters," said Sharie Haugabook, a project manager in the NCATS Therapeutics for Rare and Neglected Diseases program.

The collaborators hope to establish new animal models for use in drug screening and evaluation before the sole source contract expires in 2023.

"The most obvious reason to study rare diseases is for the patients and families affected by them. There is a lot of research being conducted on common diseases like cancer and diabetes, but some rare diseases affect only a handful of people," Lutz said. "It doesn't matter how rare a disease is, there's still a need for research. When you add up the number of diseases in the ultra-rare disease space, it affects a significant portion of the population. We can't forget about those patients and their families. We all have to do our part to make sure that we're working in those areas."

\section{Elizabeth Doughman}

Freelance writer, New Boston, NH, USA.

e-mail:esdoughman@gmail.com

Published online: 26 August 2019 https://doi.org/10.1038/s41684-019-0392-9 